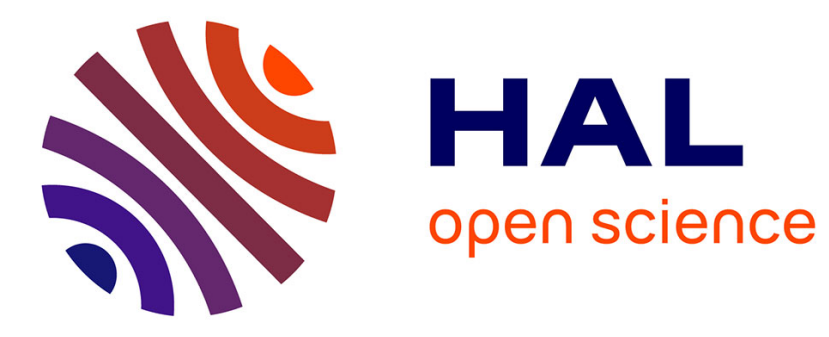

\title{
Réflexions sur la notion de champ migratoire international
}

Gildas Simon

\section{To cite this version:}

Gildas Simon. Réflexions sur la notion de champ migratoire international. Hommes et Terres du Nord, 1981, Numéro spécial, pp.85-89. 10.3406/htn.1981.1667 . hal-00609486

\section{HAL Id: hal-00609486 https://hal.science/hal-00609486}

Submitted on 19 Jul 2011

HAL is a multi-disciplinary open access archive for the deposit and dissemination of scientific research documents, whether they are published or not. The documents may come from teaching and research institutions in France or abroad, or from public or private research centers.
L'archive ouverte pluridisciplinaire HAL, est destinée au dépôt et à la diffusion de documents scientifiques de niveau recherche, publiés ou non, émanant des établissements d'enseignement et de recherche français ou étrangers, des laboratoires publics ou privés. 


\title{
RÉFLEXIONS SUR LA NOTION DE CHAMP MIGRATOIRE INTERNATIONAL
}

\author{
Gildas SIMON ${ }^{1}$
}

Dans l'optique adoptée ici, le champ migratoire est l'ensemble de l'espace parcouru, pratiqué par les migrants. La notion de champ fait référence à un espace spécifique, structuré par des flux importants, significatifs ; appliquée au domaine international, elle englobe à la fois le pays de départ et le pays d'emploi.

\section{1 - DIFFICULTÉS DE L'APPROCHE GLOBALE}

Cette approche globale mise au point dans le cas de migrations internes (1) a été peu utilisée dans le domaine des mouvements internationaux. Elle s'y heurte, en effet, à un certain nombre d'obstacles d'ordre pratique et théorique :

- les difficultés de raccordement de sources. Les recensements effectués dans les pays d'arrivée ou d'emploi ne permettent pas de connaître précisément le lieu de naissance et de résidence du migrant dans le pays d'origine, son itinéraire géographique, social ou professionnel ; inversement, les renseignements fournis par les sources statistiques du pays d'origine ignorent généralement les lieux de fixation, le devenir professionnel ou familial à l'étranger. De ce fait, tout le système de liens géographiques et socio-professionnels au niveau régional et local, de correspondances entre l'espace de départ et l'espace d'arrivée ou de fixation, échappe presque complètement à l'investigation et complique singulièrement la tâche du chercheur par rapport à l'analyse d'un champ migratoire interne. L'enquête directe reste possible dans le cadre d'un groupe restreint, d'une communauté de village dont on peut suivre les déplacements, les itinéraires et les points de fixation à l'étranger; c'est la démarche suivie par Rocha Trindade (2) par l'étude de migrants portugais entre leur village du Nord du Portugal et Orsay dans la banlieue parisienne. La connaissance du champ migratoire international d'un groupe régional ou national suppose nécessairement le recours à des sources statistiques précises et actualisées, qui sont généralement localisées dans les pays d'emploi, par exemple: titres de travail ou de séjour (3), fiches consulaires par les Tunisiens en France (4). Le caractère confidentiel de ces sources rend souvent leur accès très difficile. L'importance de la population traitée, la complexité des itinéraires migratoires entraînent obligatoirement la mise au point d'un traitement informatique approprié. On pourrait ajouter que le flou, le sous-enregistrement qui entourent la connaissance des flux internationaux tant

\footnotetext{
${ }^{1}$ Professeur de géographie, MIGRINTER - Université de Poitiers / CNRS : UMR 6588
} 
dans les pays d'origine que dans les lieux d'emploi renforcent encore les difficultés de ce type d'étude.

- sur le plan théorique, il faut mettre en cause l'approche traditionnelle de ces mouvements qui privilégie soit le point de vue de l'émigration, soit plus fréquemment celui de l'immigration. Cette démarche unilatérale, fractionnée, bloque évidemment l'analyse globale d'un champ migratoire. Sur un plan plus général, si l'économie des migrations internationales entre Etats inégalement développés est assez bien connue (5), par contre, les implications spatiales de ces flux économiques et humains n'ont pas été suffisamment approfondies. En bref, une théorie des champs migratoires internationaux reste encore à élaborer. La recherche présentée ici, à travers le cas des travailleurs tunisiens en France (4) et quelques travaux récents n'a pas la prétention d' élaborer cette théorie, elle voudrait seulement ouvrir quelques pistes pour la recherche en ce domaine.

\section{2 - PERTINENCE DE LA NOTION DE CHAMP MIGRATOIRE INTERNATIONAL}

On sait qu'un certain partage affecte l'espace migratoire international et que des courants spécifiques unissent tel pays de départ à tel pays d'emploi, les Maghrébins s'orientent dans leur grande majorité vers la France et secondairement vers le Benelux et l'Allemagne fédérale. Celle-ci constitue par contre le lieu d'emploi privilégié des Turcs, des Grecs et des Yougoslaves . Il arrive aussi que les travailleurs d'un pays, l'Italie par exemple, se répartissent de manière plus équilibrée entre la France, la Suisse et le R.F.A.

Chacun des champs migratoires créés par les relations de travail a sa spécificité car il n'affecte en réalité qu'une partie du territoire des deux pays concernés par la migration.

Dans le cas de la Tunisie, les flux dirigés vers la France s'alimentent essentiellement dans trois régions: le Sud-Est (Gouvernorats de Gabès et de Médenine), le Tell du Nord-Ouest (Jendouba, Le Kef). Ce dispositif migratoire se différencie nettement du champ migratoire interne et du champ orienté vers la Libye qui s'étend surtout sur les régions centrales du pays (Kairouan, Kasserine, Gafsa). On peut observer le même partage du pays de départ dans l'émigration espagnole avec l'opposition entre la côte de Levant polarisée par la France et les régions de l'intérieur (Andalousie) et du N.W. (Galice) drainées par l'Allemagne .

A l'intérieur du pays d'emploi, si les travailleurs étrangers sont "logés" globalement dans le même espace, une analyse approfondie montre que chaque groupe national utilise ou n'utilise qu'une partie seulement de cet espace "immigré". Les Tunisiens se regroupent plus fortement que les autres groupes étrangers à l'intérieur de trois régions: la côte méditerranéenne à l'Est de Marseille, la région lyonnaise et surtout l'agglomération parisienne. Cette spécificité du champ effectivement pratiqué se retrouve à l'intérieur même de chaque grande ville. Dans le cas de Paris analysé par M. Guillon (6), les Espagnols très nombreux dans les quartiers ouest de la capitale sont remplacés en banlieue occidentale par les Portugais, cependant que les Maghrébins se fixent essentiellement dans les quartiers nord de Paris et dans les communes ouvrières qui les prolongent dans les Hauts de Seine (pour les Marocains) et en Seine-StDenis (pour les Algériens et les Tunisiens). 
Mais on peut se demander si cette notion de champ migratoire établi selon un dispositif spécifique dans les deux pays reste encore fonctionnelle malgré l'arrêt des flux de départ et la politique d'incitation au retour qui est pratiquée par le pays d'emploi depuis la crise économique de 1973-1974. On pourrait supposer que le champ migratoire va disparaître ou se trouve amputé, restreint au pays d'immigration, en raison même du blocage de toute émigration. Ce n'est pas, en fait, le cas général.

Il faut observer que les migrations internationales de travail se poursuivent et même s'amplifient vers les Etats dotés de la rente pétrolière et dépourvus de réserves suffisantes de main d'œuvre : le nombre de travailleurs étrangers employés en Libye, en Arabie Saoudite et dans les pays du Golfe est passé de 1, 5 million e n 1975 à 2 millions en 1980 (source B.I.T.). Leur rayon de recrutement s'est considérablement élargi et s'étend désormais aux pays asiatiques de plus en plus éloignés (Pakistan, Inde, Corée du Sud).

Par ailleurs, à l'intérieur de la Communauté Economique Européenne, le traité de Rome garantit la liberté de circulation aux ressortissants des pays signataires. Les régions déshéritées de l'Italie du Sud continuent à envoyer des migrants en France et surtout en Allemagne fédérale.

On ne peut donc conclure prématurément à l'arrêt complet de l'alimentation de champs migratoires internationaux.

Par ailleurs, les mouvements de retour définitif restent relativement limités, leur intensité varie e $\mathrm{n}$ raison du degré de développement, et ne semblent pas être très supérieurs aux flux qui existaient avant le retournement du contexte migratoire en 1973-1974, et qui comprennent, de toute façon, un certain nombre de travailleurs ayant terminé leur vie active ( $1 / 3$ dans le cas tunisien).

Dans leur grande majorité, les travailleurs étrangers demeurent dans les pays d'emploi malgré la crise du marché de l'emploi, la dégradation de leurs conditions de vie et la précarité croissante de leur statut (cf. les récentes lois françaises sur l'immigration).

Cette faiblesse des retours définitifs tient moins à l'intégration des immigrés dans la société du pays d'emploi qu'aux difficultés de la réinsertion économique et sociale dans le pays d'origine et aux disparités qui persistent et souvent s'accroissent entre les deux pôles du champ migratoire (plus l'écart grandit, plus le mouvement de retour est limité).

Nombre de migrants s'installent durablement ainsi dans un statut ambigu car partagé entre leur lieu d'emploi et leur région d'origine, avec laquelle ils conservent des liens très étroits. Un nouveau mode de vie se développe, ponctuée par des retours réguliers au pays au moment de congés annuels ou à l'occasion d'évènements familiaux. Les travailleurs étrangers pratiquent ainsi des navettes à long rayon d'action à l'intérieur d'un espace de travail et de vie étiré sur plusieurs centaines et plusieurs milliers de kilomètres. Tout en essayant d'améliorer leur insertion dans le pays d'emploi, ils continuent à investir dans leur région et leur pays natal (modernisation de l'habitat, achat de commerces, placements immobiliers, etc... ). Cette situation transforme les régions de départ en banlieues éloignées des grandes villes étrangères (7).

A notre sens, cette évolution des migrations internationales, cette permanence du travail à l'étranger sans rupture de lien avec la société de départ doivent modifier l'approche des 
champs migratoires qui était fondée jusqu'à présent sur la connaissance des flux de départ annuels, et sur le nombre de travailleurs immigrés. Elle doit conduire à prendre en compte la mobilité résidentielle à l'intérieur du champ, l'intensité de la circulation aux moments des congés annuels, et chercher à apprécier l'ensemble des liens qui continuent à unir les lieux de départ aux régions d'immigration. Cette optique débouche nécessairement sur l'analyse de la pratique du champ migratoire et de sa perception par les travailleurs étrangers. Ce dernier point reste pratiquement inconnu; sauf erreur, aucune étude n'a été consacrée à ce sujet jusqu'à présent. Pourtant les interrogations ne manquent pas quant à la perception du pays d'emploi avant le départ, le mode de diffusion de cette image, les modifications de la perception du champ migratoire apportées par la migration elle-même; par exemple, le séjour à l'étranger n'a-t-il pas modifié l'image du pays d'origine ? Cette évolution ne joue-t-elle pas un certain rôle sur les intentions de retour définitif?

\section{3 - CHAMP MIGRATOIRE ET RÉSEAUX DE RELATIONS}

On retiendra d e l'optique adoptée ici qu'un champ migratoire est fondamentalement un espace de relations et qu'il faut l'étudier en tant que tel

"Nous centrons moins notre regard sur le transfert de population que sur la relation entre deux espaces (de départ et d'arrivée) que ce transfert crée. Mais cette relation ne lie pas seulement deux espaces, elle lie aussi en même temps deux formations socio-économiques" (8).

Effectivement, les migrations de travail s'inscrivent dans une série de rapports géographiques, historiques et politiques économiques et socioculturels entre les Etats concernés par la migration et c'est la spécificité de ces rapports (toujours marqués du sceau de la dépendance) qui explique la spécificité du champ géographique où s'inscrit la migration.

Ce type de rapport d'Etat à Etat est bien connu de même que l'influence des liens personnels, familiaux qui unissent le migrant à son lieu d'origine. Par contre, le rôle du groupe social, groupe villageois ou régional, fondé sur le sentiment d'appartenance et de solidarité est souvent ignoré ou sous-estimé. Pourtant le rôle de ces réseaux est fondamental dans l'organisation sociale et spatiale de ces espaces de relations internationales.

L'étude du cas tunisien (4) a permis de repérer plusieurs types de réseaux fonctionnant sur la base de l'appartenance à un groupe régional. Une typologie peut en être dressée selon le degré de cohésion de la société d'origine, son aptitude à pratiquer et à maîtriser un espace migratoire plus ou moins étendu et complexe.

Le réseau des Telliens du Nord-Ouest n'a pas dépassé le premier stade de l'évolution. Le champ de déplacement est peu étendu, inscrit essentiellement entre la Tunisie du Nord et la côte méditerranéenne française. L'espace migratoire est souvent réduit à deux ou trois pôles par exemple, Le Sers - Aix en Provence, Ghardinaou - Ajaccio - Berre dans la banlieue marseillaise. Cette pratique de l'espace est le fait de groupes ruraux longtemps enclavés, sans traditions migratoires internes (d'où faible utilisation du relais tunisois). Les possibilités d'insertion professionnelle et urbaine en France sont très limitées, ce qui explique souvent la localisation des migrants dans des petites villes et dans les campagnes françaises.

Les Tunisiens du Sud possèdent des réseaux beaucoup plus évolués qui leur permettent d'associer dans leur champ migratoire de grandes agglomérations (Tunis, Lyon, Paris) et de s'insérer dans des espaces économiques et sociaux beaucoup plus complexes que leurs 
compatriotes du Nord. Sur le plan professionnel, ils associent aux emplois traditionnels des travailleurs étrangers en milieu urbain les activités commerçantes (épiceries, pâtisseries et restaurants) qui expriment la grande capacité de ces groupes à adapter à l'extérieur les schémas mis au point par des siècles de migrations intérieures . Cette longue tradition migratoire, l'habitude de la grande ville, la cohésion et la plasticité de ce groupe humain donnent à ce réseau une assise spatiale, une stabilité dans le temps, une capacité d'adaptation aux situations difficiles (notamment depuis 1974) que les autres groupes ne possèdent pas.

Le cas des travailleurs originaires de la façade urbanisée de Bizerte à Sfax, est le plus complexe. On hésite ici plus qu'ailleurs à parler d'un réseau, il y a en réalité une multitude qui s'entrecroise. Cette diversité révèle la grande variété de ces microsociétés, qui ont conservé un sens aigu de leur identité, mais aussi les différences profondes de cette société littorale qui est confrontée à une urbanisation rapide et au développement d'activités nouvelles (industries, tourisme international). Le haut degré de scolarisation, la pratique constante du milieu urbain dans le pays d'origine donnent à ce groupe de côtiers la possibilité de pratiquer un espace beaucoup plus varié et en définitive plus étendu que les deux autres groupes régionaux de migrants.

C'est la solidité et l'articulation de ces réseaux de relations qui donnent à un champ migratoire sa cohésion profonde, qui expliquent souvent sa spécificité par rapport à l'espace pratiqué par un autre groupe national qui lui permettent de s'adapter avec plus ou moins de souplesse à la situation nouvelle créée par la crise économique et le retournement des politiques migratoires menées par les pays d'emploi.

Gildas SIMON

Université d e Poitiers

\section{BIBLIOGRAPHIE}

(1) Courgeau, Daniel (1970) Les champs migratoires en France, Paris, INED, PUF (Travaux et Documents. Cahier ; 58). $160 \mathrm{p}$.

(2) Rocha-Trindade, Maria Beatriz (1973) Immigrés portugais : observation psychosociologique d'un groupe de Portugais dans la banlieue parisienne, Orsay, Lisbonne, Instituto superior de ciências sociais e politica ultramarina, $154 \mathrm{p}$.

(3) Kolodny, E. (sous la dir.) (1977) RCP 397. Les étrangers à Stuttgart, Paris, CNRS, 315 p.

(4) Simon, Gildas (1979) L'espace des travailleurs tunisiens en France. Structures et fonctionnement d'un champ migratoire international. Thèse d'Etat, Poitiers, $426 \mathrm{p}$.

(5) Tapinos, Georges Photios (1974) L'économie des migrations internationales, Paris, Armand Colin et FNSP, 287 p.

(6) Guillon, Michelle (1978) La répartition géographique des étrangers dans l'agglomération parisienne, Aspect INSEE. Ile de France, pp.45-96.

(7) Drain, M. et Kolodny, E. (1980) L'exil pour métier : bilan de l'émigration de la Province de Séville vers l'étranger de 1960 à 1976, Paris, CNRS (travaux de la RCP ; 397).

(8) Almeida, C. (1974) Espaces socio-culturels et migrations, Colloque sur les Travailleurs Etrangers en Europe occidentale, Paris. 